\title{
Respuesta al tratamiento con lamivudina en pacientes con hepatitis crónica por virus B
}

\author{
M. D. MAÑAS GARCÍA, F. DOMPER BARDAJÍ1, A. HERNÁNDEZ ALBÚJAR ${ }^{1}$, \\ B. PIQUERAS ALCOL ${ }^{1}$, P. CARPINTERO BRIONES ${ }^{1}$ \\ Servicio de Medicina Interna y ${ }^{\prime}$ Unidad de Digestivo. Complejo Hospitalario. \\ Ciudad Real
}

\section{RESUMEN}

Objetivo: Descripción de la respuesta bioquímica y virológica en pacientes $\mathrm{HBeAg}$ positivo y negativo tratados con lamivudina. Como objetivo secundario, se valoró las modificaciones en la función renal de los pacientes tratados con este fármaco.

Método: Se estudió de forma retrospectiva a treinta pacientes con hepatitis crónica por virus B que habían realizado tratamiento con lamivudina entre noviembre de 1999 y marzo de 2004, en la Unidad de Digestivo del Complejo Hospitalario de Ciudad Real.

Resultados: Se incluyeron finalmente 29 pacientes, de los cuales 8 eran $\mathrm{HBeAg}$ positivo y $21 \mathrm{HBeAg}$ negativo. La media de duración del tratamiento con lamivudina fue de 23,7 meses (3-46). Siete pacientes habían recibido tratamiento previo con IFN. En ocho casos, se inició tratamiento posterior con adefovir. Ninguno de los pacientes del estudio negativizó el HBsAg. Entre los pacientes $\mathrm{HBeAg}$ positivo un $50 \%$ presenta actualmente valores negativos de ADN y un $25 \%$ ha negativizado el $\mathrm{HBeAg}$, aunque sin seroconversión anti-HBe. Entre los pacientes $\mathrm{HBeAg}$ negativo, un $76 \%$ han disminuido o negativizado el ADN. Observamos aparición de mutantes a la lamivudina en un 50\% y $19 \%$ de los pacientes $\mathrm{HBeAg}$ positivo y negativo respectivamente, siendo la duración media del tratamiento de dos años. Los niveles de ADN viral negativos, se asociaron en todos los casos con niveles normales de transaminasas. Los pacientes no sufrieron modificaciones en la función renal.

Conclusión: La respuesta de nuestros pacientes a la lamivudina es similar a la descrita en la literatura excepto en la tasa de seroconversión anti-HBe, que en nuestro caso ha sido nula y en el porcentaje de aparición de mutantes a la lamivudina en los pacientes $\mathrm{HBeAg}$ negativo, que en nuestro estudio ha sido menor de la esperada (19\%; $25 \%$ referido a pacientes con más de un año de tratamiento).

PALABRAS CLAVE: Lamivudina. Adefovir. Hepatitis crónica B. HBeAg.
TREATMENT RESPONSE TO LAMIVUDINE IN CHRONIC HEPATITIS B PATIENTS

\section{ABSTRACT}

Objective: The main objetive was to find a biochemistry and virology response in positive and negative $\mathrm{HBeAg}$ patients who had been treating with lamivudine. Furthermore, the secondary objetive was to made a description about kidney function changes that could be found in patients under treatment with lamivudine.

Method: Thirty patients with chronic B hepatitis under treatment with lamivudine had been retrospectively studied since November 1999 to March 2004, in a Digestive Unit, in Ciudad Real Hospital.

Results: Twenty nine out of 30 patients were included, 8 out of 29 were positive HBeAg, and 21 out of 29 were negative HBeAg.

Seven patients had been treated with IFN. Thus, 8 patients had been in need of adefovir treatment after lamivudine trial. The average of treatment had been 23.7 months ( 3 months- 46 months). In fact, none of them were changed from positive $\mathrm{HBs} \mathrm{Ag}$ to negative HBsAg. Nevertheless, we have observed those results after lamivudine treatment.

Firtsly, $50 \%$ positive $\mathrm{HBe}$ Ag patients have carried on negative DNA results. Although $25 \%$ positive $\mathrm{HBe} \mathrm{Ag}$ patients had conversed to negative $\mathrm{HBe}$ Ag. But they did not show any anti $\mathrm{HBe}$.

Secondly, 76\% negative HBe Ag patients had been suffering a DNA decreased, even though some of them had had a negative DNA results.

Thirstly, we found some lamivudine mutation $(50 \%$ in possitive $\mathrm{HBe}$ Ag patients and $19 \%$ in negative HBe Ag patients).

Finally, all the patients with negative DNA had maintained a normal AST and ALT levels. Thus, function renal had been normal under lamivudine trial.

Conclusion: According to several authors, the response to lamivudine treatment had been adequate in our population. Nevertheless, our study have shown that antiHBe levels had not been suffering any change during lamivudine treatment.

In addition, we have found a smaller figures (19\%) of lamivudine mutations in negative HBe Ag patients than other studies $(25 \%$ in patients who were followed up for 1 year).

KEY WORDS: Lamivudine. Adefovir. Chronic hepatitis B. HBeAg.

Mañas García MD, Domper Bardají F, Hernández Albújar A, Piqueras Alcol B, Carpintero Briones P. Respuesta al tratamiento con lamivudina en pacientes con hepatitis crónica por virus B. An Med Interna (Madrid) 2005; 22: 485-488. 


\section{INTRODUCCIÓN}

La hepatitis crónica por virus B representa un importante problema de salud a nivel mundial. Actualmente se calcula que existen 400 millones de personas infectadas por este virus. En nuestro país supone la tercera causa de afectación hepática crónica, precedida por la hepatitis $\mathrm{C}$ e ingesta enólica (1). Entre un 15-40\% de los pacientes infectados presentan un elevado riesgo de desarrollo de cirrosis, descompensación hepática y carcinoma hepatocelular (2).

Los principales objetivos para el tratamiento de la hepatitis crónica B son conseguir la supresión de la replicación viral de forma sostenida y la remisión de la enfermedad hepática. Esto se objetiva mediante la normalización de los niveles de ALT, niveles indetectables de ADN viral, pérdida de $\mathrm{HBeAg}$ con ó sin detección de anti-HBe y mejora en la histología (2).

El primer fármaco utilizado para su tratamiento fue el interferón alfa (IFN). Posteriormente, se aprobó la utilización de lamivudina y recientemente de adefovir divipoxil. De ellos la lamivudina en monoterapia se considera actualmente la mejor opción terapéutica para los pacientes con hepatitis crónica por virus $\mathrm{B}(3)$.

El objetivo principal del presente trabajo es la descripción de la respuesta bioquímica y virológica en pacientes $\mathrm{HBeAg}$ positivo y negativo tratados con lamivudina. Como objetivo secundario, se valoró las modificaciones en la función renal de los pacientes tratados con este fármaco.

\section{CASOS APORTADOS}

Estudio retrospectivo de 30 pacientes con hepatitis crónica por virus B tratados con lamivudina en la Unidad de Digestivo del Complejo Hospitalario de Ciudad Real, desde Noviembre del 1999 hasta marzo de 2004. La dosis de este fármaco fue de $100 \mathrm{mg}$ /día por vía oral.

Se elaboró un protocolo para la recogida de datos en el que se incluyeron la filiación, peso, talla, edad, vía de contagio, antecedentes de hepatitis aguda, presencia de otras enfermedades concomitantes, consumo de alcohol y tabaco, VHD, VHC, VIH, si había recibido tratamiento previo con IFN, grado de inflamación y estadio de fibrosis en la biopsia hepática y datos analíticos basales (antes de iniciar el tratamiento con lamivudina) entre los que se incluyeron valores de hemoglobina, leucocitos, plaquetas, actividad de protrombina, AST, ALT, urea, creatinina, alfa-fetoproteína, albúmina, colesterol total, bilirrubina, albúmina, ferritina y gammaglobulinas. En las revisiones en consulta durante el tratamiento se recogieron los niveles de creatinina, urea, ALT, HBsAg, HBeAg, y la carga viral de ADN. Se contabilizaron los pacientes con resistencia a la lamivudina que fueron tratados con adefovir. Diferenciamos los pacientes HBeAg positivo y negativo.

Los valores bioquímicos se obtuvieron mediante un analizador multicanal. Los valores normales en nuestro laboratorio son: urea (20-50 mg/dL), creatinina (0,5-1,1 mg/dL), ALT (10-40 U/L), AST (5-37 U/L). La detección de antígenos y anticuerpos se realizó mediante enzimoinmunoanálisis (ELISA). Los niveles de ADN hasta mayo de 2003 se analizaron mediante una técnica de hibridación con una sensibilidad de $3.000 .000 \mathrm{copias} / \mathrm{ml}$. A partir de esa fecha, la técnica utilizada también por hibridación molecular (Quantiplex HBV DNA; bDNA, Chiron diagnostics) tuvo una sensibilidad de 2.000 copias/ml, y desde entonces se consideró como negativa la muestra con carga viral < 100.000 copias $/ \mathrm{ml}$.

Se recogieron varios tipos de respuesta, bioquímica si se producía normalización de las transaminasas, virológica si los niveles de ADN eran indetectables con la técnica inicial de hibridación $\mathrm{O}$
$<100.000$ copias/ml con la técnica utilizada a partir de mayo de 2003 (en pacientes HBeAg positivo se valoró además la negativización del HBeAg y la aparición de anti-HBe para hablar de respuesta virológica completa), y respuesta sostenida (RS) definida como respuesta virológica seis meses tras la suspensión del tratamiento.

La biopsia hepática percutánea se realizó con aguja de tipo Menghini modificado. Los hallazgos histológicos valorados fueron el grado de inflamación y el estadio de fibrosis. Se nominaron como leve, moderado o severo.

Los datos se recogieron mediante revisión de la historia clínica y entrevista con el paciente.

Se utilizó el programa SPSS 10 para el análisis estadístico. Se realizó un análisis descriptivo de las variables incluidas en el estudio.

Se valoraron 30 pacientes HBsAg positivo, que habían sido tratados o estaban en tratamiento con lamivudina por presentar niveles elevados de transaminasas y ADN VHB positivo en suero.

De ellos 21 (72\%) eran varones. Tenían una edad media de 46,7 años (19-74), el índice de masa corporal fue de 27,6 (21,5-39,8). La vía de contagio era desconocida en 27 casos $(90 \%)$, en $2(6,7 \%)$ se debió a pinchazo accidental y en $1(3,3 \%)$ fue por transmisión perinatal. Ningún paciente tenía antecedentes de adicción a drogas por vía parenteral. Tres pacientes tenían antecedentes de hepatitis aguda. La serología para VIH, VHC y VHD fue negativa en todos los casos. Dos pacientes presentaban ingesta de alcohol de aproximadamente 20 gramos/día. En 10 casos presentaban otras patologías concomitantes, entre ellas destacaban diabetes mellitus, hipertensión arterial y tres pacientes tenían síndrome de Down.

Los valores referentes a los niveles analíticos basales de leucocitos, hemoglobina, plaquetas, actividad de protrombina, AST, ALT, urea, creatinina, alfa-fetoproteína, albúmina, colesterol total, bilirrubina, ferritina y gammaglobulinas, así como las cifras de ADN VHB se recogen en la tabla I.

Siete pacientes habían recibido tratamiento previo con IFN. Dos pacientes eran HBeAg positivo y habían recibido 9 millones de unidades/tres veces por semana durante 4 meses. Cinco pacientes eran HBeAg negativo y habían sido tratados con 6 millones de unidades/tres veces por semana durante 12 meses. Ninguno respondió al tratamiento con este fármaco, persistiendo niveles elevados de transaminasas y ADN positivo. Al revisar la respuesta de

\section{TABLA I}

VALORES ANALÍTICOS BASALES MEDIOS DE LOS PACIENTES CON HEPATITIS B CRÓNICA EN TRATAMIENTO CON LAMIVUDINA

\begin{tabular}{lc}
\hline Hemoglobina (g/dL) & $15,2(9,2-16,8)$ \\
Leucocitos/mm3 & $10.192(1300-12.000)$ \\
Plaquetas/mm3 & $193.821(61.000-347.000)$ \\
AST (UI/L) & $125(23-779)$ \\
ALT (UI/L) & $234(34-1.604)$ \\
Bilirrubina total (mg/dL) & $1,1(0,2-4,6)$ \\
Colesterol total (mg/dL) & $180(81-247)$ \\
Albúmina (mg/dL) & $4,4(2,3-4,5)$ \\
Gammaglobulinas (g/dL) & $1,5(0,7-7,2)$ \\
Actividad de protrombina (\%) & $86(42-100)$ \\
Ferritina (mg/mL) & $246(35-1.809)$ \\
Alfafetoproteína (mg/mL) & $111(2-2122)$ \\
Urea (mg/dL) & $41(24-55)$ \\
Creatinina (mg/dL) & $1,3(1-1,9)$ \\
HBsAg positivo & $29(100 \%)$ \\
HBeAg & Positivo: $8(27,6 \%)$ \\
ADN VHB (copias/ml) x 106 & Negativo: $21(72,4 \%)$ \\
\hline
\end{tabular}


este subgrupo de pacientes a la lamivudina, consiguieron respuesta virológica un paciente $\mathrm{HBeAg}$ positivo (50\%) aunque con persistencia del HBeAg y dos pacientes $\mathrm{HBeAg}$ negativo (40\%). La determinación del $\mathrm{HBeAg}$ fue negativa en 21 pacientes y positiva en 9.

La duración media del tratamiento fue de 26 meses en los HBeAg positivo (4-41) y de 23 meses en los HBeAg negativo (3-46). Siete pacientes $\mathrm{HBeAg}$ positivo y $16 \mathrm{HBeAg}$ negativo han estado más de un año en tratamiento.

Dentro del grupo de pacientes con $\mathrm{HBeAg}$ positivo, uno se perdió en el seguimiento. De los ocho restantes, tres pacientes continúan en tratamiento con lamivudina con valores normales de transaminasas y ADN negativo, e incluso uno de ellos ha negativizado el $\mathrm{HBe}-$ $\mathrm{Ag}$; en otro paciente se suspendió el tratamiento tras conseguir respuesta bioquímica y virológica, aunque con persistencia del $\mathrm{HBeAg}$; tras seis meses de seguimiento no ha habido recidiva; y los cuatro restantes se encuentran en tratamiento actual con adefovir por aparición de mutantes a la lamivudina, con reaparición de niveles elevados de ALT y ADN positivo, por encima de los valores basales. Estos últimos pacientes habían estado con lamivudina más de un año, con una media de 27 meses. Respuesta virológica completa no ha tenido ninguno de estos pacientes. Dos pacientes negativizaron el $\mathrm{HBeAg}$ aunque sin formación de anti-HBe, uno de ellos se encuentra en tratamiento con adefovir por elevación posterior del ADN sin reaparición del HBeAg y el otro, ya comentado, se encuentra en tratamiento con lamivudina para suspender ésta si persiste la respuesta durante otros seis meses como recomiendan los consensos de tratamiento (Tabla II).

\section{TABLA II}

RESPUESTA BIOQUÍMICA Y VIROLÓGICA DE PACIENTES HBeAg POSITIVO Y NEGATIVO EN TRATAMIENTO CON LAMIVUDINA

\begin{tabular}{lcc}
\hline Parámetros & HBeAg positivo & HBeAg negativo \\
\hline$N^{0}$ de pacientes & 8 & 21 Normali- \\
zación de transaminasas & $4(50 \%)$ & $7(33,3 \%)$ \\
Negativización del ADN & $4(50 \%)$ & $16 *(76,2 \%)$ \\
Negativización del HBeAg & $2(25 \%)$ & \\
Aparición de anti-HBe & $0(0 \%)$ & \\
Aparición de mutantes a lamivudina & $4(50 \%)$ & $4(19 \%)$ \\
Respuesta sostenida a los 6 meses & $1(12,5 \%)$ & $1(4,76 \%)$ \\
Negativización del HBsAg & $0(0 \%)$ & $0(0 \%)$
\end{tabular}

* Nueve de estos sólo disminución del ADN respecto al basal, sin llegar a negativizar.

De los 21 pacientes $\mathrm{HBeAg}$ negativo, 14 están recibiendo tratamiento con lamivudina, 5 de ellos presentan niveles de ADN positivos, aunque inferiores a los valores basales, y 9 negativos. En cuatro pacientes se cambió el tratamiento a adefovir por aparición de mutantes a la lamivudina. Estos pacientes llevaban más de un año en tratamiento con lamivudina, con una media de 33 meses. Valorando solo a los pacientes con más de un año de tratamiento con lamivudina, la aparición de resistencias fue del $25 \%$. Dos pacientes presentaron respuesta bioquímica y virológica al final del tratamiento, uno de ellos presenta respuesta sostenida (seis meses tras la suspensión del tratamiento) y el otro paciente ha presentado recidiva a los 4 meses de la suspensión. Un paciente con respuesta virológica falleció en lista de espera de trasplante hepático tras diagnosticarle un hepatocarcinoma. Ninguno de los pacientes del estudio ha negativizado el HBsAg (Tabla II).
Los niveles de DNA viral negativos, independientemente de la técnica utilizada, se han asociado en todos los casos con niveles normales de transaminasas. Se realizó biopsia hepática en 10 casos. El grado de inflamación fue leve en 4 casos y moderado en 6 casos. El grado de fibrosis fue leve en 5 casos y moderado en los otros 5 .

Los pacientes no sufrieron modificaciones en la función renal. Ningún paciente presentó efectos secundarios a la medicación.

En ocho casos, se inició tratamiento con adefovir. Cuatro pacientes eran $\mathrm{HBeAg}$ positivo y cuatro $\mathrm{HBeAg}$ negativo. Como ya se ha comentado, todos llevaban más de un año de tratamiento y la media de meses con lamivudina antes del inicio de resistencias fue de $27 \mathrm{y}$ 33 meses respectivamente. Un paciente ha seroconvertido con negativización del HBeAg y aparición de anti-HBe. El ADN ha disminuido en todos los pacientes, siendo indetectable en cinco de ellos tras pocos meses de tratamiento.

\section{DISCUSIÓN}

La necesidad de realizar tratamiento depende del curso de la enfermedad. Se suele recomendar en los pacientes que presentan niveles elevados de ALT, ADN viral mayor de 105 copias/ml y fibrosis moderada-grave en la biopsia, ya que son estos pacientes los que presentan una historia natural con mayor probabilidad de evolución a cirrosis.

El IFN obtiene muy buenos resultados en los pacientes que presentan niveles elevados de ALT y niveles bajos de ADN viral pre-tratamiento. En el resto de los casos los resultados son más pobres. Los frecuentes efectos secundarios dificultan el tratamiento, motivando en muchos casos el cambio de terapia (4). La lamivudina es un análogo de los nucleósidos aceptado por vía oral para el tratamiento de la hepatitis crónica por virus B. Actúa suprimiendo la replicación viral, mejora los niveles de transaminasas y la histología hepática, consiguiendo en muchos casos la seroconversión HBe. Produce muy pocos efectos secundarios, por lo que se utiliza de elección en pacientes candidatos a trasplante y con enfermedad hepática descompensada. Su respuesta es similar en pacientes con el virus salvaje o mutante precore. Al igual que ocurría con el IFN, se obtiene mejor respuesta en pacientes con mayor elevación basal de transaminasas. En nuestro estudio, en ningún caso se produjo la negativización del HBsAg. En los estudios revisados se recoge una negativización menor del 1\% (2). Un $25 \%$ de nuestros pacientes presentaron negativización del HBeAg pero ninguno de ellos tuvo seroconversión con aparición de anti-HBe. Este último dato difiere con lo encontrado en la literatura, en la que se recogen al año de tratamiento, cifras de seroconversión de un 16-18\% alcanzando en un estudio el $32 \%$ (2,7). Estos porcentajes aumentan además con la duración del tratamiento. En el $38 \%$ de los casos, se consiguieron niveles de ADN negativos. Estos resultados son similares a los recogidos en otros estudios $(2,7)$. Sólo en dos casos $(6,9 \%)$ se produjo respuesta sostenida tras seis meses de suspensión de la medicación.

Se ha demostrado que después del sexto mes de tratamiento suelen aparecer resistencias por la mutación del gen de la polimerasa en el VHB $(1,2,4,5)$. Después de tres años de tratamiento, sólo un tercio de los pacientes continúan en remisión debido a la aparición de mutantes con resistencia a la lamivudina (6). La mayor parte de nuestros pacientes han tenido tratamiento durante más de un año. En los HBeAg positivo aparecieron mutantes en un $50 \%$ de los casos. En los $\mathrm{HBeAg}$ negativo, a pesar de que la mayor parte de ellos llevaban más 
de dos años de tratamiento, solo se detectaron resistencias en un $19 \%$, cifra inferior a la publicada en la literatura.

Con respecto a los efectos secundarios, la tolerancia a la lamivudina fue buena. En el estudio de Dienstag y cols. (7), en el que compararon lamivudina y placebo en pacientes HBeAg positivo, la frecuencia de efectos secundarios fue similar en ambos grupos. En nuestro estudio no se recogieron de forma exhaustiva los efectos adversos por tratarse de un estudio retrospectivo, pero los valores analíticos de urea y creatinina no se modificaron de forma significativa en ningún paciente durante el tratamiento.

Podemos concluir que el tratamiento con lamivudina es bien tolerado y que no produce modificaciones significativas en la función renal medida con las cifras de urea y creatinina. La respuesta al tratamiento obtenida en nuestros pacientes es similar a la descrita en la literatura, excepto en la tasa de seroconversión HBeAg y en la aparición de resistencias a la lamivudina en pacientes $\mathrm{HBeAg}$ negativos que han sido menores. El adefovir puede ser la futura terapia de elección, ya que produce pocos efectos secundarios, obtiene una buena respuesta virológica y en estudios a dos años no se han observado resistencias al fármaco. Su elevado coste exige de todas formas confirmar en estudios prospectivos estos resultados.

\section{Bibliografía}

1. Buti M. Actualización en el tratamiento de la hepatitis crónica B. Gastroenterol Hepatol 2004; 27: 55-57.

2. Lok AS, McMahon BJ. Chronic hepatitis B. Hepatology 2001; 34: 12251236.

3. Ito K, Tanaka Y, Orito E, Hirashima N, Ide T, Hino T, et al. Predicting relapse after cessation of lamivudine monotherapy for chronic hepatitis $\mathrm{B}$ virus infection. Clin Infect Dis 2004; 38: 490-495.

4. Ganem D, Prince AM. Hepatitis B virus infection-natural history and clinical consequences. N Engl J Med 2004; 350: 1118-1129.

5. Suzuki F, Tsubota A, Arase Y, Akuta N, Hosaka T, Someya T, et al. Efficacy of lamivudine therapy and factors associated with emergence of resistance in chronic hepatitis B virus infection in Japan. Intervirology 2003; 46: 182-189.

6. Hadziyannis SJ, Papatheodoridis GV, Vassilopoulos D. Treatment of HBeAg negative chronic hepatitis B. Semin Liver Dis 2003; 23: 81-88.

7. Dienstag JL, Schiff ER, Wright TL, Perrillo RP, Hann HW, Gooodman $\mathrm{Z}$, et al. Lamivudine as initial treatment for chronic hepatitis B in the United States. N Engl J Med 1999; 341: 1256-1263. 